\title{
The mesoscopic modeling of laser ablation
}

\author{
A.M. Stoneham ${ }^{1}$, M.M.D. $\operatorname{Ramos}^{2}$, R.M. Ribeiro ${ }^{2}$ \\ ${ }^{1}$ Centre for Materials Research, Department of Physics and Astronomy, University College London, London WCIE 6BT, UK \\ ${ }^{2}$ Departamento da Fisica, Universidade do Minho, Braga, Portugal
}

Received: 21 July 1999/Accepted: 11 September 1999/Published online: 22 December 1999

\begin{abstract}
It is common to look at the atomic processes of removal of atoms or ions from surfaces. At this microscopic scale, one has to understand which surface ions are involved, which excited states are created, how electrons are transferred and scattered, and how the excitation leads to ion removal. It is even more common to look at continuum models of energy deposition in solids, and at the subsequent heat transfer. In these macroscopic analyses, thermal conduction is combined with empirical assumptions about surface binding. Both these pictures are useful, and both pictures have weaknesses. The atomistic pictures concentrate on relatively few atoms, and do not recognize structural features or the energy and carrier fluxes on larger scales. The continuum macroscopic models leave out crystallographic information and the interplay of the processes with high nonequilibrium at smaller scales. Fortunately, there is a middle way: mesoscopic modeling, which both models the key microstructural features and provides a link between microscopic and macroscopic. In a mesoscopic model, the length scale is determined by the system; often this scale is similar to the grain size. Microstructural features like grain boundaries or dislocations are considered explicitly. The time scale in a mesoscopic model is determined by the ablation process (such as the pulse length) rather than the short time limitations of molecular dynamics, yet the highly nonequilibrium behavior is adequately represented. Mesoscopic models are especially important when key process rates vary on a short length scale. Some microstructural feature (like those in dentine or dental enamel) may absorb light much more than others; other features (like grain boundaries) may capture carriers readily, or allow easier evaporation, or capture and retain charge (like grain boundaries); it is these processes which need a mesoscopic analysis. The results described will be taken largely from the work on $\mathrm{MgO}$ of Ribeiro, Ramos, and Stoneham for ablation by sub-band gap light.
\end{abstract}

There are several quite distinct ways to think of the target in laser ablation. One is macroscopic, when one uses standard engineering ideas on a small scale: heat supply from the laser, thermal conduction, evaporation, and perhaps thermal stress. Another way is atomistic, when one asks about which ions are removed, the electronic excited states involved, and the processes by which laser energy ends as ionic kinetic energy. The link between these two extremes is less obvious. For very many systems, it is essential to recognize an intermediate, mesoscopic, scale. At this level, the target's microstructure becomes important, and it will change as ablation proceeds. The mesoscopic scale provides a means to link macroscopic and atomistic (see, e.g., [1]); at the same time, mesoscopic methods offer a way to model complex nonequilibrium behavior in ablation.

What goes into a mesoscopic model involves a mixture of ideas. First, the computing methods are either Monte Carlo or some finite element or finite difference technique. This part is conventional. Second, there will be some continuum ideas, especially for the less critical parts of the system, and these will include standard approaches to thermal and electrical transport. Third, there will be input from the many spectroscopic and similar experiments addressing behavior at the atomic scale. Related to this will be the wealth of theoretical understanding of the defects and defect processes, including especially behavior in excited states. It is not sufficient to understand behavior close to equilibrium. Fourth, there will be information about the target microstructure. This information may be simplified to some degree, but the main features will be modeled at the level which is appropriate.

A very important point is that laser-induced processes are hierarchical. The thermal, electronic, and structural changes produced by the early stages of the pulse modify the response of the later stages. This is true even when, as in this paper, we do not consider the effects of plume generation. The modifications arise from free carrier generation, charge trapping, recombination releasing energy which creates defects or causes evaporation, and the effects of electric and stress fields. For photon energies greater than the gap, absorption is strong and is relatively uniform across the surface. For photon energies less than the band gap, absorption is concentrated 
at defect structures, including dislocations, grain boundaries, the external surface, and any impurity clouds associated with them. In nonmetals, for any bandwidth the subsequent processes are often concentrated at these defect structures. Some of these structures develop as the surface recedes during ablation, e.g., kinks on surface ledges move or dislocations climb. Others structures respond to stress, as in spalling, or to electric fields. In complex materials, especially biomaterials like teeth, there is a wider range of degradation mechanisms associated with the variety of structural components. Many key effects relate to microstructure, and lie between atomistic and continuum descriptions.

Types of microstructure. To be specific, we shall discuss simple ceramics, e.g., MgO. We shall be concerned with three main types of microstructure. First, there is the surface itself, which will be rough, and which will have on it ledges, kinks, and other defects. The roughness can vary greatly, and is very sensitive to the origin and preparation of the material. Surface energies provide some tendency toward the formation of specific crystal facets; such faceting also affects evaporation energies. Second, there are dislocations and grain boundaries. Third, there will often be inclusions of some distinct substance, such as a carbonate, hydroxide, or other oxide [2].

Imagine an idealized crystal consisting of cubic grains $5 \mu$ across. Then each cubic centimeter will contain $2 \times 10^{9}$ grains; the cubes will have an area of $3000 \mathrm{~cm}^{2}$, which corresponds to about $3 \times 10^{18}$ atoms at the grain surfaces. Even for these rather small grains, the numbers are not large. However, they are not large because they are deceptive. It is true that the width of a grain boundary from atomistic models [3] is similar to the interatomic spacing. But our concern is with optical absorption associated with grain boundaries, and optical absorption can occur readily at the defect or impurity atmospheres which are normally associated with dislocations or grain boundaries. We need not be surprised at effective thicknesses of tens of nm, similar to Debye-Hückel screening lengths. The role of dislocation absorption for sub-gap excitation has been demonstrated rather clearly [4].

For simple systems, like $\mathrm{MgO}$, mesoscopic models of ablation can use either observed grain structures or simulated microstructures. The approach can exploit both atomistic modeling and the properties of the oxide, which are known from other experiments. The same strategy should work for the more complex structures in dental materials, since the microstructure is on a similar scale. For dental enamel, the structure is based on crystallites and the ways their orientations change throughout the structure. While the structure of dentine is more complex than $\mathrm{MgO}$, and the range of processes will be greater, the same approach is possible. Almost all systems have microstructures that can influence ablation significantly, especially for biological materials $[5,6]$.

Consequences of microstructure. Dislocations and grain boundaries have roles in excitation and subsequent stages of ablation. They can be special sites for energy absorption, for carrier capture, for electron or ion transport, or for ready emission of ions. Our model [7-10] for $\mathrm{MgO}$ excited by sub-band gap light exploits a number of critical ideas. First, optical absorption of sub-band gap light occurs largely in special regions: at the surface itself, at grain boundaries, and at defects. Second, the carriers that are excited can move, be ex- cited further by the laser light, recombine at the defect sites, or be emitted from the surface. Third, there will be carrier motion, either diffusive or drift under the electric fields which evolve. Excitation from one site and recombination at another is a means of energy transport. Fourth, there will be evaporation of electrons and of ions, and this will depend on the local temperatures and binding energies. Reasonable estimates of most of the parameters are possible from experiment and can be improved by the linking of the parameters to fuller atomistic models. The ablation processes cannot be modeled fully at either the macroscopic scale (integrating heat transport equations) or at the atomistic level (as in molecular dynamics). It is crucial to include simulations at some intermediate scale.

\section{Processes in the solid state}

Our mesoscopic approach addresses three main classes of process: energy absorption, energy transfer, and evaporation. In certain applications, a further stage of diagnostics, or monitoring, requires additional processes. The mathematical methods are essentially finite difference or finite element methods, plus discrete versions of the conventional transport and conduction equations. Full details of the parameters and of the model are given in references [7-10]; in the present paper, we emphasize the physical ideas which underlie the model. The physical parameters can be varied over a range around values estimated from existing experiments. This allows a sensitivity analysis. Thus, our previous results suggest that for realistic values of parameters, the ablation behavior is not especially sensitive to the cohesive energy for surface atoms or the electron affinity of electrons.

Our model keeps track of carrier generation and carrier transport processes, as well as heat transport and ion and electron losses from the surface. It is possible to keep track of electrons with nonthermal energy distributions. The boundary conditions relate both to the material properties (periodic boundary conditions are appropriate in some cases) and to the laser beam (e.g., its space and time profiles). The mesoscopic analysis can be embedded in a larger- scale finite element model, if need be, as perhaps in a model of a whole tooth. The evolution of energy and charge in the mesoscopic region is followed throughout the laser pulse and for as long afterwards as proves necessary. Our experience for $\mathrm{MgO}$ [7-10] suggests that very long runs are needed only rarely. As the region changes in response to the laser, electrons are excited (leaving electronic holes or defect traps); there will be diffusion of heat and of excitons; and there will be charge redistribution as electrons diffuse or move under the electric fields which are generated. The electrons that have been excited are allowed to cool by interaction with lattice vibrations, to be heated by interaction with the laser beam, or to recombine with electronic holes or with traps and release heat. Some electrons will leave the solid, either as photoelectrons or by thermal evaporation (which process dominates depends on the system, and especially on the transient free electron concentration). Ionic or molecular species will leave the surface at a rate determined by the energy input to the surface. Once they have left the surface, the atoms may again interact with the laser beam or with electrons released. Many of these processes were considered 
in our earlier ceramic ablation model $[9,10]$, and we believe most processes can be handled.

Clearly, our approach goes beyond simple thermal models. These purely macroscopic descriptions assume that all energy deposited becomes thermal energy very quickly, within $0.1 \mathrm{ps}$, that a temperature is established rapidly, and that energy flows can be estimated by the use of standard thermal constants. Such models deal only with one part of the energy flow and do not distinguish between different types of energy (electronic, elastic, electronic potential/kinetic, vibrational, and defect formation energy). Even when the thermal picture gives a semiquantitative description of what is seen experimentally, this picture is very limited, and cannot be linked easily to atomic-scale models based on electronic structure [11]. The link to electronic studies is relatively straightforward with a mesoscopic model, since the basic processes correspond.

Energy absorption processes. Sub-band gap light in a transparent region (e.g., 5-eV photons in $\mathrm{MgO}$ ) will be absorbed primarily at defects or by two-photon transitions. We are then led to ask: Where will this be absorbed in the very first stages, before the plume is established and before excited electrons become significant? Without going into details, the result of an examination of a wealth of data, which is not always very well controlled, gives these good zero-order rules. First, individual defects and impurities absorb with a cross section that is typically around $0.1 \AA^{2}$. Defect and impurity densities vary greatly, but it is common to have $100 \mathrm{ppm}$ of impurities in $\mathrm{MgO}$ and similar commercial pure ceramic materials, including both standard impurities $(\mathrm{Fe}, \mathrm{Cr}, \ldots)$ and ones often ignored $(\mathrm{H}, \mathrm{C})$. For a $10-\mathrm{mJ}$ laser pulse of $100-\mathrm{fs} \mathrm{du}-$ ration (a pulse length chosen solely for illustration so that issues associated with subsequent processes may be avoided), there are about $10^{16}$ photons incident in the first $10 \mathrm{fs}$. The penetration depth is about 1000 layers, so about $10^{13}$ photons are absorbed in each layer in $10 \mathrm{fs}$, about $1 / 100$ of the number of atoms. But we should not forget the changes induced by the early part of the laser pulse, since excitation makes subsequent excitation easier. Second, grain boundaries can be regarded as regions with high densities of "defects". As noted, the effective width can easily be $10 \mathrm{~nm}$; an assumption of $10 \%$ defects in this region (ignoring the atomicscale inhomogeneity) seems acceptable. The small, highly disordered region where the grain boundary meets the surface is regarded as still more absorbant, with an effective $100 \%$ density of defects (an effective band gap less than the photon energy). Third (and similarly), surfaces should be regarded as having relatively high effective defect concentrations. In our models, we have regarded the surface (a thin layer, much more than a monolayer, but small compared with grain dimensions) as equivalent to $10 \%$ defects. Steps and kinks (especially those kink sites which capture electrons) and possibly segregated or adsorbed species can be important. In semiconductors and oxides like $\mathrm{MgO}$, laser ablation seems to involve vacancy multiplication: Vacancies already on a surface act as nuclei for further vacancy formation [12]. Vacancies can form at the edge of a cluster of vacancies in the outer surface layer, or on the second layer that has been exposed. The relative probabilities of these two processes, together with the rates of subsequent surface migration processes, determine the way in which damage evolves. One model suggests that emission of atoms from sites neighboring a vacancy generates further sites for emission. A vacancy cluster on the surface evolves from a vacancy as its neighbor atoms are ejected, which leads to still more effective sites for atomic emission. This vacancy model of laser ablation suggests that surface vacancies enhance the emission rate and make a substantial contribution to laser ablation. The ablation rates of a number of semiconductors, determined with sub-monolayer sensitivity, are within $20 \%-30 \%$ of those determined macroscopically [13].

Fourth, where grain boundaries meet surfaces, even stronger absorption is possible. We shall assume that absorption in these small, probably disordered regions is similar to that for above-gap excitation. For oxides that exist in several forms, like $\mathrm{SiO}_{2}$, disorder reduces the band gap. Finally, it is well known that absorption of energy by the electrons which have been excited to the conduction band can be very large. It is less often appreciated that transient defects are also created in many systems, and are likely to be formed more readily in the disordered grain boundary regions or at the surface. These transient defects are likely to be important in laser damage, and may be a significant source of electrons in the conduction band.

The importance of defects is very clear from the laser damage of crystals, which differ only in surface treatment. For example, for $\mathrm{CaF}_{2}$, Reichling [14] finds that thresholds for 14-ns pulses at $248 \mathrm{~nm}$ vary from $20 \mathrm{~J} / \mathrm{cm}^{2}$ for standard polished surfaces and for steps on cleaved surfaces, up to over $60 \mathrm{~J} / \mathrm{cm}^{2}$ on the terraces of cleaved surfaces. Diamondpolished and chemo- mechanically polished surfaces show intermediate values.

Energy transfer processes. These processes, including thermal conduction, energetic electron transport followed by electron hole recombination, and exciton transport, can all be influenced by the microstructure. Thermal conduction, which is reduced by grain boundary scatter, is only a modest part of energy transfer. Electron excitation, motion, and recombination is an important mechanism. Defects and grain boundaries are especially important. Point defects can often exist in more than one charge state; for many transition metal ions in $\mathrm{MgO}$, at least three charge states are stable. In particular, grain boundaries can accumulate charge and provide longrange fields. This raises a very important point: electric fields can be extremely large. This is so even in a cubic crystal, like $\mathrm{MgO}$. For piezoelectrics like $\mathrm{ZnO}$, stress-induced fields are a further complication.

It is important to make a distinction between a field $\boldsymbol{E}(\boldsymbol{r})$ which acts over some small distance, and the energy which can be given to an ion by that field, which at most is a difference in potential, $V(\boldsymbol{r})$. One such limit on ion emission energy arises when the charge responsible for $\boldsymbol{E}$ is created by electrons being moved by the incident photons of energy $E_{p h}$. To create high-energy emissions, these photons must cause a charge to build up in a small region of radius $R$. To add another electron (or hole), one photon must be able to overcome the Coulomb barrier from charges already in the region. The limit is a charge $Q_{\max }$ when the energy $E_{p h}$ just fails to move an electron by distance $a$ across the boundary of the region, i.e., $E_{p h} \approx Q_{\max }[1 / R-1 /(R+a)]$, so $Q_{\max } \approx R^{2} E_{p h} / a$. The maximum energy that can be given to a particle of charge $Z$ leaving the surface of the region is 
$Z Q_{\max } / R$, or $Z E_{p h} R / a$. Since it is hard to imagine circumstances in which $R$ is more than a few times larger than $a$, there should be a limit on ion emission energy of a few times $E_{p h}$ for particles accelerated solely by local surface charge. This is seen in [15].

Evaporation processes at the surface. "Evaporation" is an imprecise description of the many possible processes by which atoms, ions, or electrons can be removed. It is true, phenomenologically, that there is a characteristic energy needed for atom or ion removal, although many situations will need more careful descriptions. The role of water and water-related species (notably hydroxyl) is important and not always recognized explicitly. It is for these evaporation steps that contact with atomistic models is especially important. The natural simple assumption of a single removal energy for each species is certainly simplistic, but it is adequate for answers to certain types of questions, provided that different energies are adopted for the main surface and for grain boundaries, for example. The assumption is inadequate for questions about the proportions of different charge states or for the fractions of different molecular ions.

The initial evaporation steps may not eject species with equal probabilities. This has consequences. The irradiation of $\mathrm{MgO}$ by a laser beam with fluence in the range $2-10 \mathrm{~J} / \mathrm{cm}^{2}$ causes the emission of many atoms from the surface, drilling a hole. In this fluence range, the surface becomes Mg-rich early in the laser pulse, so that photons incident in the later stage of the laser pulse can absorbed strongly, causing heating. Heating can also occur by excitation of free electrons produced by ionizing defects and metal colloids on the surface. The depth of a hole created by a $3.7-\mathrm{J} / \mathrm{cm}^{2}$ laser pulse is about $1000 \AA$. If all photons were absorbed by a layer of $100 \mathrm{~nm}$, the absorbed energy per $\mathrm{MgO}$ molecule would be about $200 \mathrm{eV}$. Since vaporization requires $0.5 \mathrm{eV}$ per molecule, there is additional energy available to increase the number of photon absorbers. The laser ablation threshold of $\mathrm{MgO}$, as detected by etch depth measurements, is about $3 \mathrm{~J} / \mathrm{cm}^{2}$ [16]. The formation of color centers and of microcracks on the surface is observed even for fluences between $1-3 \mathrm{~J} / \mathrm{cm}^{2}$. There is an incubation period for fluences below twice the ablation threshold: No visible indications of ablation are seen for the first 5 laser pulses. Defects can build up in this incubation period, and then act as photon absorbers.

\section{Results: Temporal and spatial evolution}

What emerges from our approach is that in the first few picoseconds, electrons are generated at grain boundaries in sufficient numbers to start further strong absorption. Irrespective of the precise numbers, within reasonable limits, electron evaporation occurs rapidly, long before atom evaporation is significant (Fig. 1). This is partly because, for high electron work functions, the electron concentration builds up; more light is absorbed, and the electrons heat rapidly so as to overcome the higher work function. The loss of electrons leads to electric fields, which are a major factor in carrier motion. The fields may well be large enough to cause dislocation motion. After a few tens or hundreds of nanoseconds, the numbers of atoms emitted exceed the numbers of exoelectrons, which are limited by the electric field. The predicted

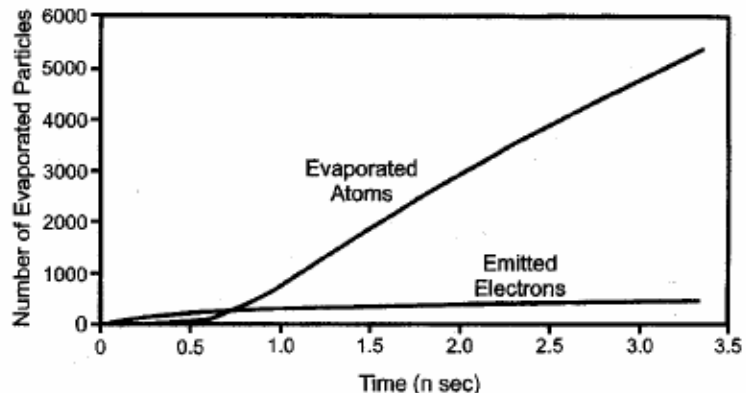

Fig. 1. Time dependence of the numbers of electrons and ions emitted (after [9])

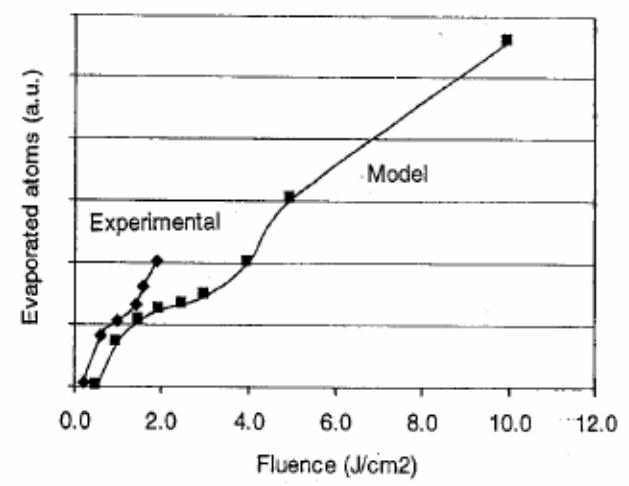

Fig. 2. Comparison of numbers of evaporated atoms predicted [10] and observed [17] as a function of fluence $\left(\mathrm{J} / \mathrm{cm}^{2}\right)$

number of evaporated atoms [10] has a dependence on laser fluence very similar to that observed [17], as shown in Fig. 2. A further conclusion from the mesoscopic modeling is that congruent removal of species is expected, since there is little dependence of emission probability for binding energies above a relatively modest value.

The inhomogeneity caused by the presence of a grain boundary shows dramatically (see Fig. 3) in the mean atomic energy (i.e., the mean kinetic energy of the atoms in the solid), the mean electronic energy, and the electric potential. This inhomogeneity has consequences. As evaporation proceeds, there is preferential loss from the grain boundaries. The removal of material depends on the angle between the laser beam and the ablating surface. This can lead to roughening and consequently to changes in absorption and removal of material; we have shown previously that this can be modeled. As is shown in Fig. 4, this leads to changes in surface roughness and ultimately to the buildup of the characteristic cone structures $[7,8]$. The extent of roughening depends on the assumed thickness of the grain boundary. "Thickness" means, of course, the width of the zone in which energy absorption occurs, and will include any defect atmosphere surrounding the boundary. The effective width will include contributions from energy absorbed by ionized centers or by electrons formed as a result of initial excitation.

We remark that completely different behavior is seen when laser irradiation is replaced by that of an electron beam. 

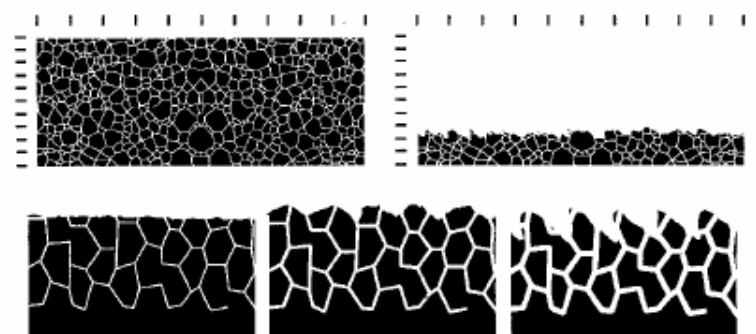

Fig. 3. Mean atomic energy (kinetic energy of atoms in the solid, in eV, shown logarithmically), electronic energy $(\mathrm{eV})$, number (per $10^{4}$ atoms) of excited electrons, and electrical potential $(V)$; the effects of the grain boundary are shown (after $[9,10])$. The data simulate results after $5 \mathrm{~ns}$ at $2 \mathrm{~J} / \mathrm{cm}^{2}$. The blocks of $\mathrm{MgO}$ have 5-nm sides. The largest fields are of order $2 \times 10^{7} \mathrm{~V} / \mathrm{cm}$, thus are approaching the breakdown field
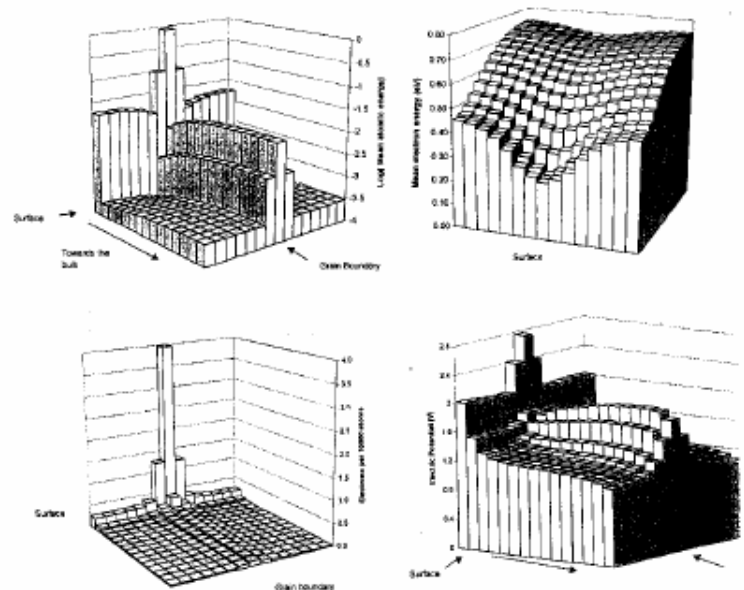

Fig. 4. Evolution of grain structure (after $[7,8]$ ) showing the consequences of roughness and of different effective grain boundary widths. The top two blocks show the evolution from an initial grain structure taken from an experimental micrograph. The lower figure shows that wider boundaries lead to rougher profiles in a striking way. The cone formation axis for the widest boundaries identifies the direction of the photon beam

The energy from the electron beam is not concentrated at defects when it is absorbed, and the rastered beam smoothes the $\mathrm{MgO}$ surface [18]. However, energy will be concentrated at defects in subsequent processes.

The evolving morphology suggests that one could choose conditions so as to optimize surface texture and roughness, e.g., in preparing a tooth for filling material, since only physical adhesion is involved. In the dental case, the nature and extent of undercutting will be the primary control. It should be possible to do neat, low-volume undercuts which will retain the filling without destroying too much good tooth.

\section{Conclusions}

What we have described are the results of our initial mesoscopic models of ablation. The approach incorporates some standard components, such as Monte Carlo and finite difference methods, and adopts (where appropriate) standard approaches to thermal and electrical transport. We have designed our approach to allow input from the many spectroscopic and related experiments on behavior at the atomic scale, and also to exploit the wealth of theoretical understanding of the defects and defect processes, including especially behavior in excited states. A primary aim has been to find a simple, yet realistic route to the inclusion of target microstructure.

It is not sufficient to understand behavior close to equilibrium. Our approach addresses naturally the fact that laserinduced processes are hierarchical. Thermal, electronic, and structural changes during the early stages of the pulse modify what happens later. This is so even when, as here, we omit the effects of plume generation. The major phenomena which contribute hierarchically include free carrier generation and charge trapping, from electron-hole or exciton recombination releasing energy, which create defects or cause loss from the surface and from the effects of electric and stress fields. For photon energies less than the band gap, absorption is concentrated at defect structures, including dislocations, grain boundaries, the external surface, and the impurity clouds associated with them. In nonmetals generally, for any bandwidth, the subsequent processes are often concentrated at these defect structures; yet the microstructure is not constant in time. Structures evolve as the surface recedes during ablation. Others structures respond to stress, as in spalling, or to electric fields, as seen in electrical breakdown studies. In complex materials, like teeth, there is an even wider range of degradation mechanisms associated with the variety of structural components. Many key effects relate to microstructure and lie between atomistic and continuum descriptions. Generalizing mesoscopic modeling to cover the broader range of phenomena seems practical. The application of these models to the optimization of ablation is a greater challenge, but appears within our grasp as the underlying physics becomes clearer.

Acknowledgements. This work was supported in part by Fundacao para a Ciencia e Tecnologia under project PBICT/FIS $/ 2151 / 95$ and by EPSRC grants GR/L02678 and GR/L70615.

\section{References}

1. T.C. Choy, J.H. Harding, A.H. Harker, P.A. Mulheran, L.W. Smith, A.M. Stoneham: In Computer Aided Innovation of New Materials, ed. by M. Doyama (Elsevier, Amsterdam 1991) p. 869, et seq.

2. A.M. Stoneham, P.W. Tasker: In Surface and Near Surface Chemistry of Oxide Materials, ed. by L-C. Dufour, J. Nowotny (Elsevier, Amsterdam 1987) pp. 1-22

3. P.W. Tasker, D.M. Duffy: Phil. Mag. A 47, 817 (1983)

4. J.T. Dickinson, L.C. Jensen, R.L. Webb, M.L. Dawes, S.C. Langford: J. Appl. Phys, 74, 3758 (1993); see also R.L. Webb, L.C. Jensen, S.C. Langford, J.T. Dickinson: J. Appl. Phys. 74, 2323 (1993) and R.L. Webb, L.C. Jensen, S.C. Langford, J.T. Dickinson: J. Appl. Phys. 74, 2338 (1993)

5. D'Arcy W. Thompson: On Growth and Form (Cambridge University Press Cambridge, UK 1917; abridged edition 1961)

6. E.A.M. Kidd, S. Joyston-Bechell: Dental caries: essentials of dental caries, In Dental Anatomy and Embryology, ed. by J.W. Osborn, p. 155-209

7. R.M. Ribeiro, Marta M.D. Ramos, A.M. Stoneham, J.M. Correia Pires: Appl. Surf. Sci. 109-110, 158 (1997) 
8. R.M. Ribeiro, Marta M.D. Ramos, A.M. Stoneham: Proc. Int. Conf Advances in Materials and Processing Technol., Vol. II (AMPT 97 Guimaraes July 1997) pp. 489-494

9. R.M. Ribeiro, Marta M.D. Ramos, A.M. Stoneham: Comput. Mater. Sci. 10, 33, (1998)

10. R.M. Ribeiro, Marta M.D. Ramos, A.M. Stoneham: MRS Proc. 526, 21 (1998)

11. N. Itoh, A.M. Stoneham: Materials Modification by Electronic Excitation (Cambridge University (unpublished))

12. A. Okano, A.Y. Matsuura, K. Hattori, N. Itoh, J. Singh: J. Appl. Phys. 71, 3158 (1993)
13. N. Itoh, A. Okano, K. Hattori, J. Kanasaki, Y Nakai: Nucl. Instrum. Methods B 82, 310 (1993); N. Itoh, J. Kanasaki, A. Okano, Y. Nakai: Ann. Rev. Mater. Sci. 25, 97 (1995)

14. M Reichling: private communication to A.M. Stoneham (1999)

15. J.T. Dickinson, S.C. Langford, J.J. Shin, D.L. Doering: Phys. Rev, Lett. 73, 2360 (1994)

16. L. Dirnberger, et al.: Appl. Surf. Sci, 69, 216 (1993)

17. H. Ishibashi, S. Arisaka, K Kinoshita, T. Kobayashi: Jpn. J. Appl Phys. 33, 4971 (1994)

18. T.J. Bullough, C.J. Humphreys, R.W. Devenish: MRS Proc. 157, 323 $(1990)$ 